\title{
Fluorescence in Situ Hybridization and Transmission Electron Microscopy in Spermatozoa from Patients with High FSH Levels
}

\author{
Giulia Collodel ${ }^{1}$, Paola Piomboni ${ }^{1}$, Alice Luddi ${ }^{1}$, Cristina Salvatici $^{2}$ and Elena Moretti ${ }^{1}$ \\ ${ }^{1}$ Department of Molecular and Developmental Medicine, University of Siena, Siena, Italy \\ ${ }^{2}$ CE.M.E, CNR, Via Madonna del Piano,10, 50019 Sesto Fiorentino, Firenze, Italy
}

Received: December 09, 2013; Accepted: January 17, 2014, Published: January 31, 2015

"Corresponding author: Giulia Collodel, Department of Molecular and Developmental Medicine, University of Siena, Policlinico Le Scotte, Viale Bracci, 14, 53100 Siena, Italy, Tel: 39-0577-233539; Fax: 39-0577-233527; E-mail: giulia.collodel@unisi.it

\begin{abstract}
The aim of this study was to evaluate the sperm morphology and aneuploidies in patients with elevated Follicle Stimulating Hormone (FSH) levels. Spermatozoa were examined by light and electron microscopy to determine semen parameters and details of fine morphology. Fluorescence in situ hybridization (FISH) analysis was performed in order to investigate the meiotic behavior of chromosomes 18, $\mathrm{X}$ and $\mathrm{Y}$.

Sperm concentration was lower than the 25 centile in seven out of eight patients and comprised between the 50 centile and the 75 centile, in only one patient; sperm progressive motility appeared lower than the 10 centile in six out of eight patients and comprised between the 10 centile and the 25 centile in two cases. Ultrastructural sperm examination showed a severely deranged spermatogenetic process; the quantification of TEM data showed significantly higher percentages of immaturity $(P<0.01)$ and apoptosis $(P<0.01)$ and a reduced fertility index in spermatozoa from patients than those observed in sperm from controls. The FISH analysis showed in the group of patients with elevated FSH levels a diploidy rate significantly higher $(P<0.01)$ compared to controls; on the other hand, the observed rates of disomy of chromosome 18 and sex chromosome were similar to those evaluated in controls.
\end{abstract}

In conclusion, men with high serum FSH level showed sperm with ultrastructural characteristics of apoptosis and immaturity and elevated percentage of diploidies evaluated by FISH. The increased frequency of diploidy could be related to the presence of binucleated sperm or sperm with double chromosomal set, a marker of an altered maturation process or to apoptosis that cause DNA fragmentation enhancing the number of spots detected by this cytogenetic procedure.

Keywords: Aneuploidies; FISH; FSH level; Sperm; TEM

\section{Introduction}

Sperm aneuploidy screening has been used as a tool in diagnosis and determination of treatment options for male factor infertility [1], in particular it was applied in populations with asthenozoospermia [2], teratozoospermia [3], oligozoospermia [4], and with altered karyotype [5].

The chromosomal aneuploidies were analyzed even in the sperm extracted from testis with regard to the different conditions determining azoospermia including groups with nonobstructive azoospermia and in severe oligozoospermia with high Follicle Stimulating Hormone (FSH) levels [6]. To the best of our knowledge, reports showing values of sperm aneuploidies in presence of high FSH levels are lacking. On the other hand, the effect of elevated FSH levels in the development of aneuploidies in oocytes is better documented $[7,8]$.

FSH, released by the anterior pituitary gland via stimulation from gonadotrophin-releasing hormone, reflects the status of spermatogenesis and it is known for its role in the initial development of Sertoli cells and in their stimulation for spermatogonial proliferation. FSH level $>4$.5IU/L was associated with abnormal semen analysis in terms of morphology and sperm concentrations [9]. In addition, Piomboni et al. [10] suggested that, in men with idiopathic infertility, normal FSH levels and an elevated frequency of total sperm aneuploidy, deranged meiotic segregation could be reduced by human recombinant FSH treatment (150 IU on alternate days for 3 months), suggesting a possible relationship between FSH level and segregation of chromosomes. Because individuals with abnormal semen parameters make up the majority of intracytoplasmic sperm injection (ICSI) candidates, the investigation of the chromosomal constitution of their spermatozoa is of great interest. In this study, we analyzed sperm quality from selected patients with elevated FSH levels in serum. Semen samples were examined by light and electron microscopy. TEM data were elaborated with a mathematical formula able to indicate a fertility index and the presence of the three main sperm pathologies: apoptosis, immaturity, and necrosis. Meiotic chromosome segregation was investigated by FISH carried out on sperm nuclei, using probes for chromosomes 18, $\mathrm{X}$ and $\mathrm{Y}$.

\section{Materials and Methods}

\section{Patients}

Among patients addressed at the Centre for Diagnosis and Treatment of Couple Sterility, Siena University, between 2010- 
2012, 25 individuals showed elevated levels of FSH and/or LH and normal testosterone. Among them, 17 were excluded from this study; six out of 25 were azoospermic, five had varicocele, four urogenital infections, one was cryptorchid, one showed globozoospermia, a sperm defect of supposed genetic origin. In the end, we obtained eight individuals (aged 30-43 years) who did not show or report any other pathologies potentially responsible for hypergonadotropic hypogonadism. Patients were interviewed about their case histories, reproductive problems and family backgrounds. They had a normal 46, XY karyotype evaluated by conventional cytogenetic analysis, no history of diabetes, radiotherapy, chemotherapy, chronic illness or medications. Microbiological investigation of semen specimens and urethral fluids excluded the presence of genitourinary infections. Clinical and physical examinations and scrotal Eco-color Doppler were performed in all patients to detect the possible presence of varicocele or other anatomical problems. All patients showed a BMI $<25 \mathrm{~kg} / \mathrm{m}^{2}$. Serum hormone levels were evaluated using chemiluminescence performed with a commercial kit (Beckman Coulter Access for FSH, LH and Testosterone). Normal ranges were: $0.7-11.00 \mathrm{mU} / \mathrm{ml}$ for FSH (sensitivity $0.2 \mathrm{mUI} / \mathrm{ml}$, intraand inter-assay coefficient of variation $<10 \%$ ), 0.8-8.0 mU/L for LH (sensitivity $0.2 \mathrm{mUI} / \mathrm{ml}$, intra- and inter-assay coefficient of variation $<10 \%$ ) and $2.7-10.9 \mathrm{mg} / \mathrm{ml}$ for testosterone (sensitivity $0.1 \mathrm{ng} / \mathrm{ml}$, intra- and inter-assay coefficient of variation < 10\%). All patients provided written informed consent before inclusion in this study.

\section{Light microscopy of semen}

Semen was collected by masturbation after 4 days of sexual abstinence and examined after liquefaction for $30 \mathrm{~min}$ at $37^{\circ} \mathrm{C}$. Volume, pH, sperm concentration and motility were evaluated according to WHO [11] guidelines. In patients with a sperm concentration of less than 10 million/ml, PCR analysis on DNA extracted from blood lymphocytes (QIAamp DNA Blood kit, QIAGEN, Milan, Italy) was carried out to exclude the presence of Y chromosome microdeletions.

\section{Transmission electron microscopy of semen}

For electron microscopy, sperm samples were fixed in cold Karnovsky fixative and maintained at $4^{\circ} \mathrm{C}$ for two hours. Fixed semen was washed in $0.1 \mathrm{~mol} / \mathrm{l}$ cacodylate buffer ( $\mathrm{pH}$ 7.2) for 12 hours, postfixed in $1 \%$ buffered osmium tetroxide for one hour at $4^{\circ} \mathrm{C}$, then dehydrated and embedded in Epon Araldite. Ultra-thin sections were cut with a Supernova ultramicrotome (Reickert Jung, Vienna, Austria), mounted on copper grids, stained with uranyl acetate and lead citrate and then observed and photographed with a Philips EM208 electron microscope (Philips Scientifics, Eindhoven, The Netherlands).

For each patient, three hundred ultra-thin sperm sections were analyzed. Major submicroscopic characteristics were recorded by two trained examiners who were blind to the experiment. TEM data was evaluated using the statistical mathematical formula by Baccetti et al. [12]. This formula considers 16 selected submicroscopic characteristics of sperm organelles able to define sperm function and calculates the number of spermatozoa probably free of structural defects (Fertility Index, FI) and the percentages of three main phenotypic sperm pathologies: immaturity, necrosis and apoptosis [13], each one characterized by typical alterations of organelles. Reduced acrosomes, round or elliptical nuclei with uncondensed chromatin, and the presence of cytoplasmic droplets were the examined characteristics for immaturity. Marginated chromatin and altered shaped nuclei were considered a main ultrastructural markers of apoptosis, whereas spermatozoa with broken plasma membranes, reacted acrosomes, and disrupted chromatin are affected by necrosis. Sperm characteristics from 25 men of proven fertility were used as reference values [13].

\section{FISH analysis of spermatozoa}

To evaluate aneuploidy frequency, FISH was performed according to [14] in the sperm nuclei of all the patients. A combination of $\alpha$-satellite DNA probes (CEP, Chromosome Enumeration Probes, Vysis, IL, USA) for chromosomes 18, X, and Y, directly labeled with different fluorochromes, was used. Observation and scoring were performed using a Leitz Aristoplan Optical Microscope (Leica, Wetzlar, Germany), equipped with a fluorescence apparatus with a triple bandpass filter for aqua, orange and green fluorochromes (Vysis), and a monochrome filter for DAPI (4',6-diamidino-2-phenylindole). Semen samples from 25 fertile men (aged 22 to 40 years) were analyzed and used as controls [13]. Whenever possible, nearly 5,000 sperm nuclei were scored for each sample.

\section{Statistical analysis}

Statistical analysis was performed using the version 8 SAS system (Sas Institute Inc. Cary, NC 27513, USA). The Wilcoxon scores (rank sums) were used to compare the considered variables such as aneuploidy rates, percentages of ultrastructural sperm pathologies, and fertility index between patients and controls. The control group made up of men of proven fertility [13]. $P<0.05$ was considered significant.

\section{Results and Discussion}

The aim of this study was to evaluate the sperm morphology and aneuploidies in patients with elevated FSH levels. Spermatozoa were examined by light and electron microscopy to determine semen parameters and details of fine morphology (Table 1). FISH analysis was performed in order to investigate the meiotic behavior of chromosomes $18, \mathrm{X}$ and $\mathrm{Y}$ (Table 2). So far, the concomitant assessment of narrow investigations, such as TEM and FISH, on human ejaculated sperm allowed us to provide important information on the spermatogenetic impairment in patients with different pathologies related to male infertility [1517].

For these reasons we decided to apply these methods to spermatozoa from patients with elevated serum FSH levels. In this study, sperm concentration was lower than the 2.5 centile [11] in four out of eight patients, comprised between the 2.5 centile and the 5 centile in one out of eight men, comprised between the 10 centile and the 25 centile in two patients, and comprised between the 50 centile and the 75 centile in only one 


\begin{tabular}{|c|c|c|c|c|c|c|c|c|}
\hline \multirow[b]{2}{*}{ Patients } & \multicolumn{3}{|c|}{ Spermiogram data } & \multirow[b]{2}{*}{ FSH levels } & \multirow[b]{2}{*}{$\mathrm{FI}^{*}$} & \multicolumn{3}{|c|}{ Sperm Pathologies\% } \\
\hline & $\begin{array}{l}\text { Vol } \\
(\mathrm{ml})\end{array}$ & $\mathrm{Sp} / \mathrm{ml} \times 10^{6}$ & Motility\% & & & Apoptosis* & Immaturity* & Necrosis \\
\hline 1 & 3 & 2.3 & 30 & 15.8 & 48836 & 7.60 & 78.58 & 33.75 \\
\hline 2 & 1.4 & 6 & 26 & 15.8 & 3287 & 14.32 & 68.62 & 34.31 \\
\hline 3 & 1.3 & 87 & 40 & 13.6 & 437853 & 13.24 & 73.48 & 24.89 \\
\hline 4 & 4.5 & 36 & 12 & 16.1 & 5678 & 28.53 & 57.25 & 30.02 \\
\hline 5 & 3 & 28 & 26 & 21.5 & 342298 & 3.80 & 86.98 & 21.56 \\
\hline 6 & 7.5 & 13 & 33 & 41.8 & 98973 & 24.11 & 76.87 & 31.44 \\
\hline 7 & 2.5 & 7 & 33 & 19.9 & 72152 & 7.77 & 77.69 & 24.39 \\
\hline 8 & 4.4 & 8 & 44 & 17.6 & 135814 & 10.26 & 70.09 & 24.26 \\
\hline Mean \pm SD & & & & & $143111.4 \pm 160762.1$ & $13.70 \pm 8.55$ & $73.70 \pm 8.75$ & $28.08 \pm 4.88$ \\
\hline Normal range & [11] & [11] & [11] & $\begin{array}{l}0.7-11 \\
\mathrm{mU} / \mathrm{ml}\end{array}$ & $\begin{array}{c}07386079.7 \pm \\
10464288\end{array}$ & ${ }^{\circ} 4.06 \pm 2.05$ & ${ }^{\circ} 48.83 \pm 13.93$ & ${ }^{\circ} 32.13 \pm 10.58$ \\
\hline
\end{tabular}

Table 1: Spermiogram data, FSH levels and TEM scores obtained by mathematical elaboration of sperm ultrastructural analysis of 8 patients considered in this study.

${ }^{*} P<0.01{ }^{\circ}$ Mean percentages $( \pm \mathrm{SD}$ ) of FI and percentage of pathologies in spermatozoa from 25 healthy men of proven fertility [13].

\begin{tabular}{|c|c|c|c|c|c|c|}
\hline Patients & $\mathbf{n}^{\circ}$ cells scored & Diploidy\%* & $18 \%$ & $\mathrm{XX} \%$ & YY\% & $\mathrm{XY} \%$ \\
\hline 1 & 2549 & 0.282 & 0.080 & 0.040 & 0 & 0.040 \\
\hline 2 & 2489 & 0.514 & 0.064 & 0.064 & 0.096 & 0.193 \\
\hline 3 & 5643 & 0.573 & 0.094 & 0.119 & 0.119 & 0.215 \\
\hline 4 & 5325 & 0.324 & 0.230 & 0.139 & 0.046 & 0.186 \\
\hline 5 & 4231 & 0.508 & 0.156 & 0.039 & 0.039 & 0.078 \\
\hline 6 & 3669 & 0.410 & 0.072 & 0.070 & 0.020 & 0.170 \\
\hline 7 & 3498 & 0.477 & 0.118 & 0.149 & 0.059 & 0.089 \\
\hline 8 & 3212 & 0.439 & 0.076 & 0.077 & 0.077 & 0.194 \\
\hline Mean \pm SD & & $0.441 \pm 0.099$ & $0.111 \pm 0.057$ & $0.087 \pm 0.043$ & $0.057 \pm 0.039$ & $0.146 \pm 0.066$ \\
\hline Controls $^{\circ}$ & 128352 & $0.277 \pm 0.073$ & $0.103 \pm 0.052$ & $0.060 \pm 0.057$ & $0.048 \pm 0.032$ & $0.137 \pm 0.071$ \\
\hline
\end{tabular}

Table 2: Frequencies of sperm disomy and diploidy of chromosomes $18, \mathrm{X}$ and $\mathrm{Y}$ in eight patients with high FSH levels.

${ }^{*} P<0.01$; ${ }^{\circ}$ Mean percentages $( \pm \mathrm{SD})$ of disomy and diploidy for the examined chromosomes in spermatozoa from 25 healthy men of proven fertility [13].

patient. Sperm progressive motility [11] appeared lower than the 2.5 centile in three out of eight patients, comprised between the $5^{\text {th }}$ centile and the $10^{\text {th }}$ centile in three out of eight analyzed samples and comprised between the $10^{\text {th }}$ centile and the $25^{\text {th }}$ centile in two cases (Table 1). This data agreed with those of other studies $[9,18]$ showing the association between high FSH levels and abnormal semen parameters.

Ultrastructural sperm examination showed a severely deranged spermatogenetic process. Whereas, sperm necrosis was not diffuse in such kind of sperm, since there was only a small increase in two out of eight patients compared with the reference value obtained from semen analysis of proven fertile men (Table 1). The quantification of TEM data showed significantly higher percentages of immaturity $(P<0.01)$ and apoptosis $(P<0.01)$ in spermatozoa from patients than those observed in sperm from controls. It is noteworthy that in each analyzed specimens, the percentages of apoptosis and immaturity were elevated (except apoptosis in patient 5) in respect to that reported in the reference values (Table 1). Moreover, TEM analysis revealed in each ejaculate the presence of sperm with double nuclei (range 2-10\%, Figure 1), indicating alteration of germ cell division during spermatogenesis and sperm with marginated chromatin, feature of sperm apoptosis (Figure 2). Similar characteristics have already been described in sperm of patients with hypergonadotropic hypogonadism and with different polymorphisms in LH and FSH receptor [18]: a further evidence of the role of FSH in the spermatogenetic process $[19,20]$. The effect of FSH in regulation of apoptosis in human germinal epithelium was reported in an in vitro study where the administration of FSH and testosterone promoted cell survival and differentiation [21]. Previously, Henriksen et al. [22] had suggested that FSH plays a regulatory stage-specific function on apoptosis and DNA synthesis during spermatogenetic process of adult rats.

In the cases analyzed in this study, also the FI (number of sperm free of ultrastructural defects) obtained by TEM analysis 
mathematically elaborated was very low, demonstrating a severely reduced sperm quality.

Since majority of these patients are candidates for ICSI, the FISH analysis performed directly on sperm nuclei provides a better understanding of the meiotic process and it could improve the risk assessment of chromosomally abnormal embryos.

FISH sperm analysis was performed on the sperm nuclei of all the patients. A total of 30,616 sperm was scored, evaluating the disomy and diploidy frequencies of chromosomes $18, \mathrm{X}$ and $\mathrm{Y}$ (Table 2). Means \pm SD of FISH values obtained from 25 fertile males, used as controls, are shown in Table 2.

In the group of patients with elevated FSH levels, the diploidy rate was significantly higher $(P<0.01)$ compared to range value. On the other hand, the observed rates of disomy of chromosome 18 and sex chromosome were similar to those evaluated in sperm from controls (Table 2).

An association between high serum FSH levels and the occurrence of sperm chromosome aneuploidies has been

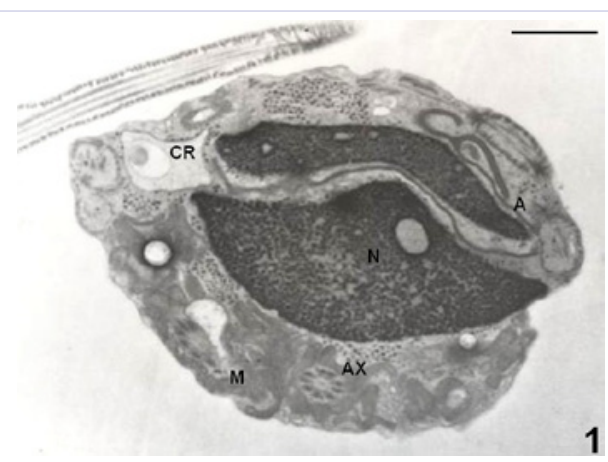

Figure 1: TEM micrograph of a binucleated sperm; nucleus (N), acrosome (A), mitochondria (M), cytoplasmic residue (CR), axoneme (AX). Bar $1 \mu \mathrm{m}$.

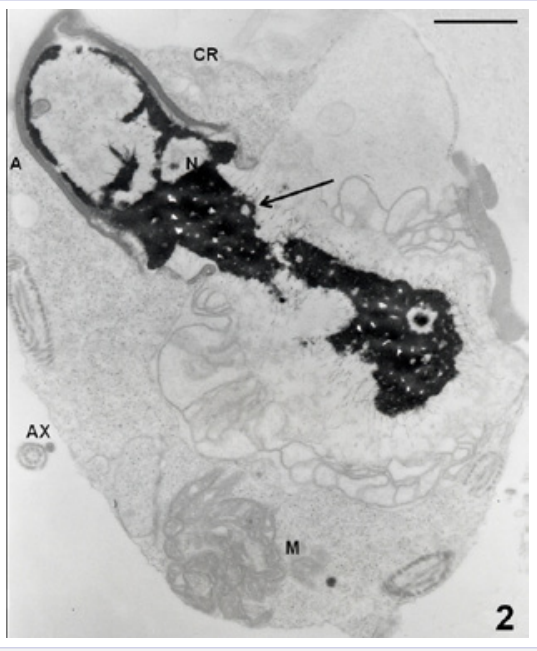

Figure 2: TEM micrograph of an apoptotic sperm. The chromatin appears marginated and fragmented (arrow); acrosome (A), mitochondria (M), cytoplasmic residue (CR), axoneme (AX). Bar $2 \mu \mathrm{m}$. reported in testicular sperm and in ejaculated sperm from oligozoospermic men even if a little number of cells were evaluated [6]. In addition, FSH affects metaphase I chromosome alignment and increases aneuploidy in mouse oocytes matured in vitro [7] and high follicular fluid FSH levels were associated with oocyte aneuploidy in women having undergone controlled ovarian hyperstimulation [23]. The high frequency of sperm diploidy, observed in this study, might indicate an incomplete meiotic process leading to immature, sometimes binucleated sperm, as highlighted by TEM examination.

An other possible explanation of the increased rate of sperm diploidy is related to the presence of apoptosis that induces significantly higher DNA fragmentation rates in human ejaculated sperm [24] and a strong link between DNA fragmentation and the presence of numerical chromosome abnormalities was also detected [25].

\section{Conclusions}

In conclusion, men with high serum FSH level showed sperm with ultrastructural characteristics of apoptosis and immaturity and elevated percentage of diploidies evaluated by FISH. The increased frequency of diploidy could be related to the presence of binucleated sperm or sperm with double chromosomal set, marker of altered maturation process or to apoptosis that cause DNA fragmentation enhancing the number of spots detected by this cytogenetic procedure.

\section{References}

1. Emery BR (2013) Sperm aneuploidy testing using fluorescence in situ hybridization. Methods Mol Biol 927: 167-173.

2. Collodel G, Capitani S, Baccetti B, Pammolli A, Moretti E (2007) Sperm aneuploidies and low progressive motility. Hum Reprod 22: 18931898.

3. Machev N, Gosset P, Viville S (2005) Chromosome abnormalities in sperm from infertile men with normal somatic karyotypes: teratozoospermia. Cytogenet Genome Res 111: 352-357.

4. Martin RH, Rademaker AW, Greene C, Ko E, Hoang T, et al. (2003) A comparison of the frequency of sperm chromosome abnormalities in men with mild, moderate, and severe oligozoospermia. Biol Reprod 69: 535-539.

5. Piomboni P, Stendardi A, Gambera L (2014) Chromosomal aberrations and aneuploidies of spermatozoa. Adv Exp Med Biol 791: 27-52.

6. Levron J, Aviram-Goldring A, Madgar I, Raviv G, Barkai G, Dor J (2001) Sperm chromosome abnormalities in men with severe male factor infertility who are undergoing in vitro fertilization with intracytoplasmic sperm injection. Fert Steril 76: 479-484.

7. Roberts R, Iatropoulu A, Ciantar D, Stark J, Becker DL, Franks S, Hardy K (2005) Follicle-stimulating hormone affects metaphase I chromosome alignment and increases aneuploidy in mouse oocytes matured in virtro. Biol Reprod 72: 107-118.

8. Dursun P, Gultekin M, Yuce K, Ayhan A (2006) What is the underlying cause of aneuploidy associated with increasing maternal age? It is associated with elevated levels of gonadotropins? Med Hypotheses 66: $143-147$. 
9. Gordestsky J, van Wijngaarden E, O’Brien J (2012) Redefining abnormal follicle-stimulating hormone in the male infertility population. BJU Int 110: 568-572.

10. Piomboni P, Serafini F, Gambera L, Musacchio C, Collodel G, et al. (2009) Sperm aneuploidies after human recombinant follicle stimulating hormone therapy in infertile males. RBM Online 18: 622-629.

11. World Health Organization (2010) WHO laboratory manual for the examination and processing of human semen. ( $5^{\text {th }}$ edn) WHO Press.

12. Baccetti B, Bernieri G, Burrini AG, Collodel G, Crisa N, et al. (1995) Notulae seminologicae. 5. Mathematical evaluation of interdependent submicroscopic sperm alterations. J Androl 16: 356-371.

13. Collodel G, Moretti E (2008) Morphology and meiotic segregation in spermatozoa from men of proven fertility. J Androl 29: 106-114.

14. Baccetti B, Bruni E, Collodel G, Gambera L, Moretti E, et al. (2003) 10 , 15 reciprocal translocation in an infertile man: ultrastructural and fluorescence in-situ hybridization sperm study: case report. Hum Reprod 18: 2302-2308.

15. Baccetti B, Bruni E, Collodel G, Mancini S, Piomboni P, et al. (2006) Studies on varicocele III. Ultrastructural studies of spermatozoa from infertile males with varicocele and 18, X and Y aneuploidies. J Androl 27: 94-101.

16. Moretti E, Di Cairano G, Capitani S, Scapigliati G, Baccetti B, et al. (2007) Cryptorchidism and semen quality: a TEM and molecular study. J Androl 28: 194-199.

17. Moretti E, Pascarelli NA, Giannerini V, Geminiani M, Anichini C, et al. (2009) 18, X, Y aneuploidies and transmission electron microscopy studies in spermatozoa from five carriers of different reciprocal translocations. Asian J Androl 11: 325-332.
18. Collodel G, Cantara S, Di Cairano G, Moretti E, Taglianetti E, et al. (2013) Alterations of the FSH and LH receptor genes and evaluation of sperm ultrastructure in men with idiopathic hypergonadotropic hypogonadism. J Assist Reprod Genet 30: 1101-1108.

19. Baccetti B, Strehler E, Capitani S, Collodel G, De Santo M, et al. (1997) The effect of follicle stimulating hormone therapy on human sperm structure (Notulae seminologicae 11). Hum Reprod 12: 1955-1968.

20. Sairam MR, Krishnamurthy H (2001) The role of follicle-stimulating hormone in spermatogenesis: lessons from knockout animal models. Arch Med Res 32: 601-608.

21. Sa' R, Neves R, Fernandes S, Alves C, Carvalho F, et al. (2008) Cytological and expression studies and quantitative analysis of the temporal and stage-specific effects of follicle-stimulating hormone and testosterone during cocultures of the normal human seminiferous epithelium. Biol Reprod 79: 962-975.

22. Henriksen K, Kangasniemi M, Parvinen M, Kaipia A, Hakovirta $H$ (1996) In vitro, follicle-stimulating hormone prevents apoptosis and stimulates deoxyribonucleic acid synthesis in the rat seminiferous epithelium in a stage-specific fashion. Endocrinology 137: 2141-2149.

23. Hammoud I, Vialard F, Bergere M, Albert M, Gomes DM, et al. (2012) Follicular fluid protein content (FSH, LH, PG4, E2 and AMH) and polar body aneuploidy. J Assist Reprod Genet 29: 1123-1134.

24. Brugnon F, Van Assche E, Verheyen G, Sion B, Boucher D, et al. (2006) Study of two markers of apoptosis and meiotic segregation in ejaculated sperm of chromosomal translocation carrier patients. Hum Reprod 21: 685-693.

25. Enciso M, Alfarawati S, Wells D (2013) Increased numbers of DNAdamaged spermatozoa in samples presenting an elevated rate of numerical chromosome abnormalities. Hum Reprod 28: 1707-1715. 